\title{
On location of service control
}

\author{
Heikki Hämmäinen and Pekka Lahtinen \\ Nokia Research Center \\ P.O.Box 45, FIN-00211 Helsinki, Finland \\ heikki.hammainen@research.nokia.fi, \\ pekka.lahtinen@research.nokia.fi
}

\begin{abstract}
This paper discusses the requirements imposed by broadband services on location of service control. Discussion is based on the early results of TINA consortium. TINA proposes a modular object-oriented control architecture which allows services to be designed with little consideration on the final deployed configuration. This flexibility promotes "free competition" between locations of service control, between CPE and telecom platforms in particular.
\end{abstract}

\section{INTRODUCTION}

TINA $^{1}$ consortium is specifying the TINA software architecture in order to speed up the harmonization of computing and telecommunications technologies [TINA-RM, 1993].

So far TINA has contributed significantly in the areas of connection management [TINACMA, 1993] and distributed processing environment (DPE [TINA-DPE, 1994]). TINA connection management is independent of transport network technology but aims at a standards impact on ATM signaling phase 3 based on separation of call and connection control. TINA DPE extends the concepts of CORBA [OMG, 1994] by for instance integrating message-based and stream-based object interfaces as well as connectionless and connectionoriented communications.

An important part of TINA is the proper organization of service control, i.e. distributed processing and storage of service logic and data. The wide range of existing telephony-based services and the difficulty of predicting the future broadband service features suggest to have functional flexibility as a high priority design goal. TINA adds flexibility for instance by pursuing location independence and decentralized control of services.

This paper proceeds bottom-up. The general technical requirements of location independent object interactions are first elaborated. Then the essence of service control objects and service

1 TINA-C stands for Telecommunication Information Networking Architecture Consortium. The circa 30 member companies of TINA-C maintain an international group of 40 researchers at Bellcore in Red Bank, New Jersey, USA. The author of this paper worked in the group during 1993. 
control architecture are reviewed. Finally some problems of flexible location of service control are discussed.

\section{LOCATION INDEPENDENCE}

By location independence we mean that potentially active objects (i.e. code and data) can be moved from one location (i.e. computing environment) to another with minimal technical and managerial overhead. External interfaces and semantics of the affected service are preserved. Only the non-functional quality of service aspects may change. This is an ambitious goal that requires common agreements on

1. processing platform

2. object language

3. common object services

4. service design guidelines.

TINA DPE platform is based on the POSIX standard and maps the application programming interfaces onto the underlying native operating systems: threads, local system calls, remote procedure calls, stubs, kernel transport service, etc. DPE facilitates portability of application software modules written in $\mathrm{C}$ and $\mathrm{C}++$.

TINA ODL (Object Definition Language) extends the OMG IDL by making a distinction between an object and its interfaces and by supporting the specification of both. ODL facilitates interoperability and portability of object and interface templates across DPE platforms. So far ODL does not, however, sufficiently cover the specification of object behavior. Therefore TINA Preprocessing Language (TPL) was developed to provide a temporary solution for easy port of TINA conformant object templates between DPE platforms. TPL is an extension to C++. TPL preprocessor takes TPL as an input and generates $\mathrm{C}++$. ODL and TPL generate $\mathrm{C}++$ stubs which support location and access transparencies [ODP, 1992]. Location transparency, i.e. late binding of object interactions, is based on removing hardcoded object references by using traders to maintain and mediate information on object locations [ODP, 1992]. Access transparency, i.e. interworking across heterogeneous object implementations, is based on automatic conversion between network's message transfer syntax and the local object's internal message representation.

TINA has produced draft specifications for a service infrastructure, i.e. a set of common object services. Object portability is significantly enhanced if an object template can assume that the same or corresponding common object services are available to it in any new location. Examples of such services are naming service, event service, object factory service, terminal mobility service, personal mobility service, etc. [TINA-SA, 1993, OMG, 1994].

TINA provides design guidelines which promote location independence in several ways. For instance, adhering to a service design process based on the ODP viewpoint model [ODP, 1992] postpones decisions on object locations up to the deployment phase. Service objects are designed and implemented on the ODP computational level with minimal assumptions on their physical locations. 


\section{SERVICE CONTROL OBJECTS}

The term service control denotes here the user perceived on-line control aspects of networking services. Examples of such services and service components are directory searches, call forwarding, tailored billing, authentication, authorization, video conference bridging, voice mail, and quality of service negotiation.

Control is implemented as logic and data. Distinction between logic and data is, however, vague and sometimes unimportant. In practice, logic can be implemented for instance as interpreted scripts, rule sets, executable binary programs, or dynamic link modules. Data may appear for instance as rows of relational tables, state variables of object instances, or less structured global variables.

TINA, like other object-driven approaches, encapsulates logic and data in objects. Logic is mostly embedded in object templates and data in object instances. It should be noted that an object instance can be activated only in an environment where its behavior description, e.g. ODL/TPL/C++ object template, is available in executable form. Further, the behavior description needs its execution environment, e.g. ODL interpreter and DPE-extended operating system, to be available and activated.

In IN terms (e.g. IN CS1 [CCITT, 1991]), service logic can be represented as a script running on Service Logic Interpreter (SLI) in Service Logic Execution Environment (SLEE) in Service Control Point (SCP). Scripts assume that SLEE contains a predefined set of functional Service Independent Building Blocks (SIB). If the script language and SIB set are standardized, scripts can be easily moved across SCP platforms. Service data is represented as (1) volatile service-specific variables called Service Instance Data belonging to a script, (2) SIB-specific managed parameters called Service Support Data, and (3) service-specific data stored in a database potentially residing on a separate Service Data Point (SDP) platform. If the representation of database schemas and query language are standardized, service data can be easily moved across SDPs.

\section{SERVICE CONTROL ARCHITECTURE}

In the IN architecture, service logic runs in servers (i.e. in SCPs) whereas access references reside in switches (i.e. in SSP trigger tables). That is, switches know where the service logic is located and how to activate it. Activation happens as a "side-effect" of calling to a given number. In the Internet architecture, terminals (i.e. application client modules) know how to find the servers running the service logic (i.e. application server modules). Some service logic may run in the terminal side, but the network (of routers) remains as a provider of plain bit pipes.

As IN takes the service control software and databases out of switches, TINA does the same to IN call processing software and trigger tables. Switches are seen as a simple switching function controlled by objects running on DPE compliant terminals and servers. Distinction between terminals and servers can be vaguely made based on differences in degree of DPE capability, processing capacity, reliability, mobility, set of stubs and objects for common object services, application objects, scope of node management, and ownership.

Designers of applications and new services can assume the existence of a set of common object services. This includes services for controling connection-oriented communication sessions such as person-to-person audio. According to TMN layered abstractions (see 
Figure 1), Element Layer consisting of network elements (e.g. switches) is controlled by Connection Managers (CM) residing on the Element Management Layer. CMs on Network Management Layer are responsible of link-by-link control thru the Element Management Layer. Session managers (SM) residing on the TMN Service Layer facilitate end-to-end negotiation between Service Access Functions (SAF) before reserving SAF-to-SAF transport resources from the lower layers. SAF is further decomposed into Terminal Agent and User Agent which represent the interests and capabilities of individual terminals (i.e. owners) and users.

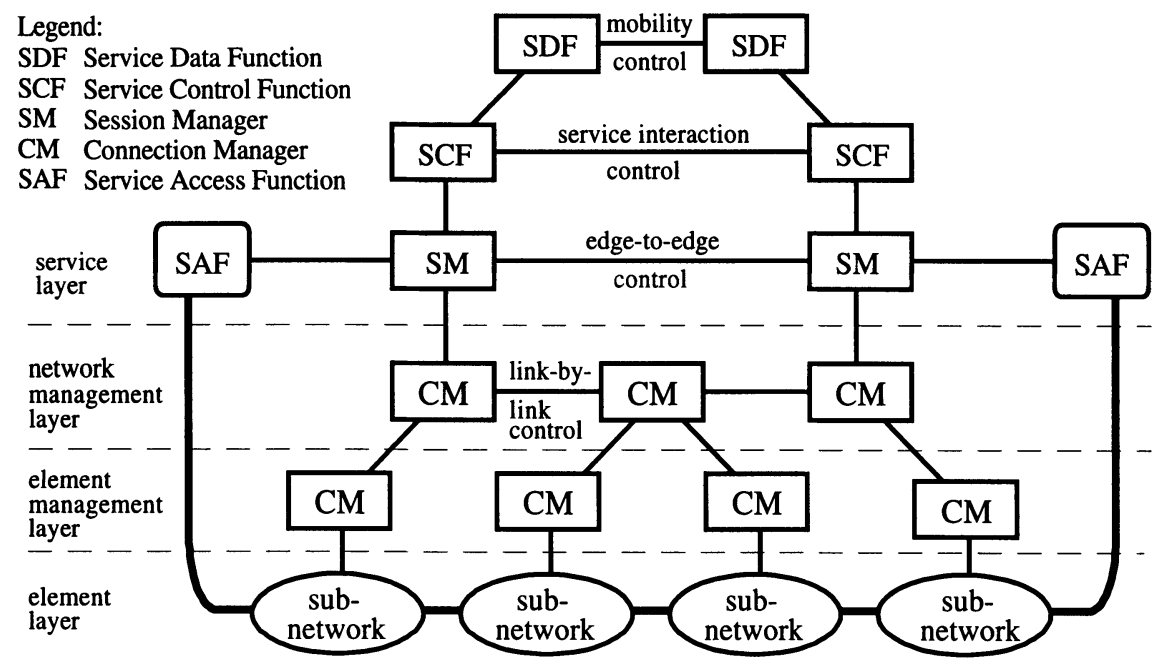

Figure 1. IN/TMN based reference model for service control.

Connections are represented as Connection Graphs (CG) where vertices represent switches, edges represent connections between switches, and ports represent end points of edges [TINA-CMA, 1993]. Each CG has a level-specific synchronized representation on each level of abstraction (i.e. Element Layer through Service Layer). For example, user-initiated changes to CG (e.g. add party to conference) are propagated top-down from Service Layer to Element Layer, and network-initiated changes to CG (e.g. bulldozer cut a wire) are propagated bottom-up from Element Layer to Service Layer if necessary. State changes of a CG need to be propagated horizontally between SMs if the participants of a CG reside in different SM domains.

CG provides a multiparty mechanism for users, or application programs, to negotiate on usage of network resources. In addition to switches, more specialized resources such as video bridges and signal converters can be controlled thru CGs. In an opportunistic scenario new types of resources can be deployed with little extensions to CG objects. The CG-based approach to session control has got positive feedback from small scale experiments performed on the Touring Machine platform [Coan et al., 1993]. 


\section{EXAMPLE}

Traditionally, two kinds of disjoint network and terminal scenarios have flourished:

- dumb terminals (like ordinary telephones) connected to an intelligent network (like an ISDN with $\mathbb{I N}$ features), network providing the services, and

- smart terminals (like computers and workstations with TCP/IP protocols and networking capabilities) connected to a dumb network (like the fixed connections onto which the Internet is based), terminals and terminal-like servers providing the services.

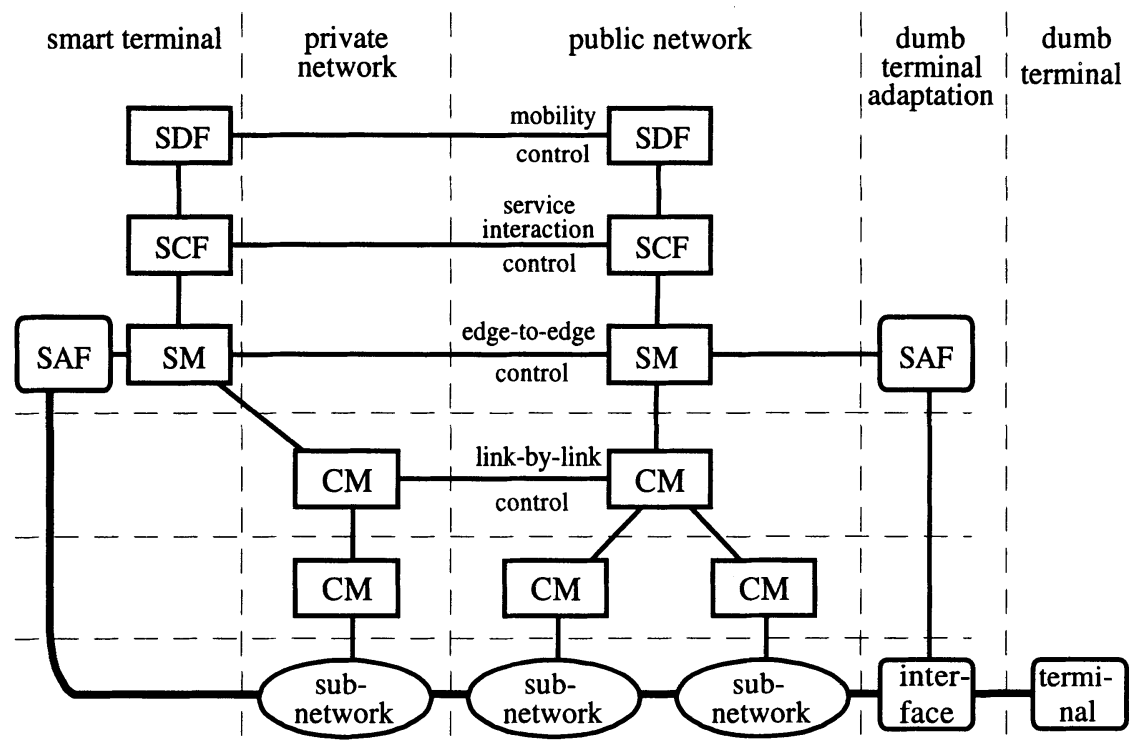

Figure 2. An example of the assignment of functionalities onto organizations and network components.

In the future, this cannot be the case any more, but the same network and terminal scenario must support various assignments of functionalities onto the terminals, network components, and participating organizations. Figure 2 illustrates one example of such a scenario.

One cannot expect that all terminals were capable of supporting any functionalities, not even protocol-based negotiation of services. Therefore the dumb terminals must be adapted to a future telecommunications architecture by including on the network side as an adaptation interface some functionalities which logically reside on the terminal itself. This is illustrated on the right side of Figure 2.

On the other hand, a smart terminal - illustrated on the left of Figure 2 - may itself perform session, service and data management (SM, SCF, SDF) for itself, in addition to the actual Service Access Funtion (SAF). Nevertheless intelligent and dumb terminals can freely interwork with each other within the same service, thanks to the adaptation - it does not matter where the functionalities are actually placed, as long as they are available for the terminals. 
Figure 2 also illustrates the fact that the telecommunications network of the future will consist of public parts (owned by telecoms operators) and private parts (owned and operated by their customer organizations), and the control and management implemented by the various participating organizations must co-operate in order to jointly provide the services.

\section{DISCUSSION}

IN currently supports telephony services based on single point of control which means that at most one service logic script in a single SCP can be active at a time for a call. TINA distributes the functionality into terminals and specialized servers. For instance, users may have personal scripts for controling incoming and outgoing calls and users may access several services potentially at the same time. This implies dealing with multiple points of control, or distributed peer-to-peer control, since it is likely that even a basic person-to-person call involves two potentially conflicting scripts.

The conventional routing and billing logic of IN is distributed into the SAF and SM functions in TINA. Both SAF and SM use available distributed common object services for various purposes. SM typically runs on shared DPE servers whereas SAF can partly run on DPE capable terminals. SAF has a modular object structure including user and terminal agents, which allows some flexibility in physical configuration of SAF objects between terminal and server platforms. Exploiting the location independence of object interactions, on-demand downloading of stubs, instances, and even templates of SAF objects is possible. In some cases, this may happen per service instantiation. For instance, a mobile call involves downloading the mobile user's personal profile from his remote home location register to the local visitor location register, or even to the mobile terminal, for local processing. It has been suggested that on-demand transfer of executable application and service logic, e.g. object templates, becomes desirable along with more powerful workstations and terminals [Hämmäinen, 1991].

Flexible location of service logic and data brings information networking services onto a new level of dynamics. This raises difficult issues concerning feature interactions, data security, service reliability, service introduction, billing, etc. For instance, the problem of unwanted feature interactions emerging in IN, see e.g. [Cameron et al., 1993], gets more complex in the case of multiple points of control. Preparing for future service features when designing the current one is difficult. Within a single server such as IN SCP it is possible to analyze a new feature against existing features and make changes if necessary. In an open network containing multiple servers managed by different service providers this is not possible. Independently designed service features are likely to interact, often with harmful consequences. Introduction of object-oriented techniques, generic negotiation protocols between servers, and user-adjustable interaction attributes are however expected to alleviate the feature interaction problem in the long term [Griffeth et al., 1992]. The fine tuning of service features must be left to users because they finally decide whether a feature interaction is harmful or not.

\section{CONCLUSION}

We have described how TINA architecture adds flexibility to the location management of service control. This flexibility is necessary to facilitate deployment and usage of alternative 
location schemes. The underlying assumption is that location flexibility leads into a more optimal location scheme for each particular usage situation. Optimal can mean for instance more efficient exploitation of distributed computing resources or more rapid introduction of new service features.

The inevitable downside of location flexibility is the increased complexity. Feature interactions are problematic in the relatively simple single point of control architecture of IN. Applying location flexibility for multiple points of control in TINA services is likely to be significantly more complex. Much experimentation and trialing is needed to make this part of TINA mature enough for commercial deployment.

\section{REFERENCES}

Cameron J., Velthuijsen H.: Feature Interactions in Telecommunications Systems: A Software Perspective, IEEE Communications Magazine, August 1993

Coan B., Gopal G., Herman G., Leland W., Mak V., Sekar R., Vecchi M., Weinrib A., Wuu S.: The Touring Machine System (Ver. 3): An Open Distributed Platform for Information Networking Applications, Proc. of TINA Workshop, L'Aquila, Italy, September 27-30, 1993

CCITT: CCITT Study Group XI, draft recommendation Q.1200 series "Intelligent Networks", Geneva, September 1991

Griffeth N., Velthuijsen H.: The negotiating agent model for rapid feature development, proc. of Software Engineering for Telecommunications Systems and Services, Florence, Italy, March/April 1992

Hämmäinen H.: Form-based Approach to Distributed Cooperative Work, PhD thesis, Faculty of Information Technology, Helsinki University of Technology, 1991

ODP: Basic Reference Model of Open Distributed Processing - Part 3: Prescriptive Model, DR X.903, ISO/IEC JTC1/SC21/WG7, November 1992

OMG: Common Object Services Specification, ed. John Siegel, The Object Management Group, March 1994

Ryan R.: The SCAI Standard, proc. of GlobeCom '93, Houston, Texas, December 1993

TINA-CMA: Connection Management Architecture, TINA-C Core Team, Red Bank, December 1993

TINA-DPE: Distributed Processing Environment, Version 0.1, TINA-C Core Team, Red Bank, January 1994

TINA-RM: TINA Architecture Rooadmap, TINA-C Core Team, Red Bank, December 1993

TINA-SA: Service Architecture, TINA-C Core Team, Red Bank, December 1993 